\title{
Apoptosomes and Proteasomes from Exosomes Generated by Human Hematopoietic Stem Cells
}

\author{
Igor Prudnikov *, Anton Smirnov and Volodymyr Tsyvkin \\ Laboratory of Stem Cell Biology, O. Bogomolets Institute of Physiology, NASU, Kyiv, Ukraine \\ * Correspondence: igor.prudnikov@biph.kiev.ua; Tel.: +38-067-625-52-20 \\ $\dagger$ Presented at the Cell-to-Cell Metabolic Cross-Talk in Physiology and Pathology, 17 December 2020 to 17 \\ January 2021; Available online: https://cells2020.sciforum.net/. \\ Published: date
}

\begin{abstract}
The activities of two classes of neutral proteases were studied: caspases and proteasomes, which could be contained in vesicles generated by human stem cells. The formation of apoptosomes could not be induced in human mesenchymal and hematopoietic stem cells (hereinafter - MSCs and HSCs, respectively) with the participation of cytochrome C. Caspase activity was found in the culture medium and in exosomes after the TNF $\alpha$ supplementation only. This activity is completely inhibited by a non-substrate caspase inhibitor emricane, and is not sensitive to proteasome inhibitors. It is assumed that this activity has a membrane and intracellular location. Supplementing of TNF $\alpha$ cell culture leads to the generation of neutrophils and other leukocytes, the formation of apoptosomes in them, which are secreted with the exosomes and remain circulating outside the cells. Extracellular activity pertained to protein complexes of molecular weight similar to $20 \mathrm{~S}$ proteasomes and is probably represented by apoptosomes. It is suggested that TNF $\alpha$ induces in HSC culture the appearance of neutrophils or the generation of other differentiated cells that are capable of apoptosis, in contrast to HSCs or MSCs.
\end{abstract}

Keywords: apoptosis; caspase; exosomes; human hematopoietic stem cells; human mesenchymal stem cells; proteasome; neutrophils; TNF $\alpha$

\section{Introduction}

Recently, the list of intercellular communicators has been supplemented by extracellular vesicles, whose content and membrane proteins are involved in cell signaling or its modulation [1]. It is known that white blood cells secrete exosomes containing proteasomes in the form of $20 \mathrm{~S}$ particles [2]. It was found that proteasomal activity is important not only for intracellular proteolysis, as proved by the presence of functional proteasomes in blood plasma, alveolar fluid, and in the culture medium of some cells [3,4]. The process of ubiquitination and the work of the proteasomal system are important for the development of tissues and organs and the maintenance of cellular homeostasis. For example, it was found that the lack of Rpt3, a protein in the regulatory subunit of proteasomes, leads to inactivation of muscle stem cell cells and their apoptosis [5]. The opposite example is ubiquitin-specific protease 7 , which deubiquitinates proteasome targets and this prevents apoptosis of leukemic cells [6]. Proteasome inhibitors cause programmed death of myelogenous leukemia stem cells, "saving" p53 protein from proteolytic degradation, but do not cause apoptosis of HSCs, as they do not contain this protein in significant quantities required for the regulatory activity $[7,8]$.

During apoptosis, caspases organize a global program of cell destruction, targeting about a thousand of protein substrates [9]. These proteases cause DNA damage, inhibit transcription, stop the translation of proteins and disable many other important cellular processes. There are a few undirect data indicating the existence of a canonical apoptotic mode of cell death in HSCs with 
formation of apoptosomes [10,11]. The traditional classification of caspases as inflammatory and apoptotic has changed over the last decade, as it has become clear that caspases involved in the inflammatory response, such as caspase- 1 , can cause cell death, and apoptotic caspases (caspase- 9 , 3 and -7$)$ can mediate processes that do not cause it $[12,13]$. Spontaneous neutrophil death mediated by caspase- 3 is a prototype of programmed cell death and is critical for modulating inflammatory responses; however, the main regulatory pathways remain poorly understood. In old neutrophils, the cleavage and activation of caspase- 3 does not depend on the canonical pathway mediated by caspase- 8 or caspase- 9 . Instead, caspase- 3 is activated by serine proteinase 3 (PR3), which is presented in the cytoplasm of these neutrophils and is released from the granules into the cytoplasm [14]. PR3 cleaves procaspase-3 at the cleavage site of caspase-9. Adaptive transfer of neutrophils with PR3 deficiency has shown that their delayed death occurs through the other internal pathways of apoptosis / survival, rather than through the inflammatory microenvironment. The presence of a suicidal inhibitor of the above protease (SERPINB1) reduces the activity of PR3 in old neutrophils, and the removal of SERPINB1 accelerates the death of these cells. PR3-mediated caspase-3 activation controls spontaneous neutrophil death, but whether this process exists in stem cells is unknown. Whether PR3 can be active in HSCs is also unknown [14,15]. Alternatively, there are other variants of cell death, such as the external pathway of apoptosis or necroptosis, and they could be related to the homeostasis of HSCs. In contrast to apoptosis, necroptosis is a regulated form of necrotic cell death mediated by receptor protein kinase 3 and mixed lineage kinase domain-like protein (MLKL) $[16,17]$. It is triggered by external ligands such as TNF $\alpha$ [16]. Stimulation of the TNF $\alpha$ receptor leads to phosphorylation of MLKL. Phosphorylated MLKL oligomerizes on cytoplasmic necrosomes and disrupts membrane integrity, leading to cell death $[17,18]$. Pyroptosis as a process mainly described for monocytes and macrophages, is caused by inflammatory caspases, such as caspase- $1,-4,-5,-11$ [19]. This form of cell death is determined by specific limited cleavage of gasdermin D (GSDMD) with release its $n$-terminal domain GsdmD p30 [20]. GsdmD p30 collects with the formation of pores in the plasma membrane, which leads to cell lysis [21].

Whether HSCs programmed cell death occurs by activating the internal apoptosis pathway and whether this process forms a stable pool of cells in the stem state remains to be determined. It is possible that programmed cell death does not exist in the HSCs in the canonical form. We investigated the activities of two classes of neutral proteases: caspases and proteasomes, which could be contained in vesicles generated by adult human stem cells and which are involved in normal cell physiology, often determining the fate of cells and their very existence. Moreover this issue is important due to the practical tasks - the usage of vesicles (mainly exosomes) as a therapeutic agent. We compared HSCs vesicles with those generated by MSCs because the last are more studied.

\section{Experiments}

Bone marrow samples from sternum of 3 healthy male volunteers 38, 44, and 54 years old were donated to isolate adult human hematopoietic stem cells after obtaining informed consent and approval of the study protocol by the Institute's Ethics Committee. MSCs and HSCs were separated from the rest of bone marrow cells by mean of a negative selection procedure with monoclonal antibodies (StemCell, USA). For performing this antibodies to the antigens CD3, CD11b, CD14, CD16, CD19, CD56, CD66b for HSC and CD3, CD14, CD19, CD38, CD66b for MSCs were applied as RosetteSep cocktails, designed to enrich the samples with progenitor cells. The isolated cells were resuspended in StemSpan ${ }^{\text {TM }}$ Serum-Free Expansion Medium (SFEM) and MesenCult ${ }^{\text {TM MSC Basal }}$ Medium supplemented with an appropriate stimulant supplement containing recombinant human cytokines: Flt3L, SCF, IL-3, IL-6 and TPO [22]. A similar medium was used for MSCs. Cells were cultured in a $95 \%$ humidified incubator at $37{ }^{\circ} \mathrm{C}$ in $5 \% \mathrm{CO}_{2}$. The medium was changed every three days during 3 weeks. The cultured cells were concentrated by positive selection for $\mathrm{CD}^{2} 4^{+}$antigen using EasySep ${ }^{\mathrm{TM}}$ Human CD34 Positive Selection Kit on magnetic particles. The isolated cells were permeabilized with saponin $50 \mu \mathrm{g} / \mathrm{mL}$ before further manipulations and stored on ice for 15-60 min in an isotonic buffer medium in a volume of $100-200 \mu \mathrm{L}$. 
Part of HSCs after 10 days of cultivation was used to induce the generation of neutrophils and cell death induction by TNF $\alpha$ SFEM medium was replaced with one containing TNF $\alpha$, IL-2 and GMCSF of $10 \mathrm{ng} / \mathrm{mL}$ (hereinafter "TNF $\alpha$ cocktail" or "TNF $\alpha$ " in the captions to the figures). Cells were grown in the new medium for 4 days.

The conditioned culture mediums were filtered through a $0.22 \mu \mathrm{m}$ filter, centrifuged for $30 \mathrm{~min}$ at $15,000 \times g$, and concentrated in 12-15 folds with $150 \mathrm{KD}$ Millipore concentrators. The concentrate was used for gel filtration and exosome isolation with the kit for "Total exosome isolation" (Invitrogen, USA). Exosomes were also isolated by centrifugation for $3.5 \mathrm{~h}\left(4^{\circ} \mathrm{C}\right)$ at $\mathrm{RCF}=50,000 \times \mathrm{g}$. The precipitate was resuspended in the isotonic medium and stored on ice.

The concentrated conditioned culture medium was fractionated by high molecular weight gel filtration with HW-65 (Supelco, Japan) on a column of $50 \times 1 \mathrm{~cm}$, at the rate of $0.3 \mathrm{~mL} / \mathrm{min}$. Each fraction was re-concentrated on 150 KD filters (Millipore, USA).

Apoptosis was induced in saponin-permeabilized cells with buffered solution of cytochrome $\mathrm{C}$ $(5 \mu \mathrm{M})$ and dATP $(1 \mathrm{mM})$ for $1 \mathrm{~h}$ at $32{ }^{\circ} \mathrm{C}$ with the addition of protease inhibitors cocktail (Sigma, USA) and $2 \mathrm{mM}$ DTT [23]. Protease reactions were performed in a volume of $200 \mu \mathrm{L}$ at $37^{\circ} \mathrm{C}$. The activity of caspase-3 was measured using the substrate (Z-Asp-Glu-Val-Asp)2-Rhodamine 110 (ZDEVD) $)_{2}$ R110 in $10 \mathrm{mM}$ Tris or $30 \mathrm{mM}$ HEPES buffer, $(\mathrm{pH} 7.3)$ at $\quad$ ex $=485 \mathrm{~nm}$ and $\quad e m=535 \mathrm{~nm}$. (ZDEVD) ${ }_{2}$ R110 as a substrate was used to measure caspase activity together with $20 \mu \mathrm{M}$ bortezomib or $2 \mu \mathrm{M}$ AdaAhx ${ }_{3} \mathrm{~L}_{3} \mathrm{VS}$ to reduce background proteasomal activity. Emricasan was added to confirm caspases' specificity. The chymotrypsin-like proteasomal activity was measured using the Z-LLYVAMC substrate in the same medium at $\quad e x=360 \mathrm{~nm}$ and $\quad e m=440 \mathrm{~nm}$. Specificity was confirmed by the addition of inhibitors of all types of proteasomal activity: bortezomib $(2 \mu \mathrm{M})$, AdaAhx ${ }_{3} \mathrm{~L}_{3} \mathrm{VS}(2$ $\mu \mathrm{M})$ or $0.1 \mu \mathrm{M}$ epoxomycin in the case of chymotrypsin-like only [23].

mRNA from cultivated cell samples was isolated to detect the expression of specific antigens. 20 $\mu \mathrm{g}$ of total RNA from each sample of HSCs tested was isolated using RNeasy mini kit (Qiagen, USA) and treated with DNase I (GE Healthcare, USA). cDNA synthesis was performed using SuperScript III reverse transcriptase (Invitrogen, USA) with polyA primers, according to the manufacturer's recommended procedure, and cDNA was treated with RNase H (Hoffmann-La Roche, USA) after synthesis. Hot-start Tfi DNA polymerase (Invitrogen, USA) was used for PCR reactions according to the manufacturer's recommended procedure. PCR reaction products of mRNAs of CD14, CD34 and CD45 protein antigens were treated with EarI restriction enzyme (Fermentas, USA) to confirm the specificity of used primers. PCR with a minus template was included in the PCR experiment as a negative control, and primers to the cDNA fragment of the $15 S$ protein of the small RNA ribosomal subunit (primers from Ambion, USA) were used as a positive control.

\section{Results}

Two cell populations were isolated and propagated from each bone marrow sample. HSCs expressed mRNAs that corresponded to the phenotype $\mathrm{CD} 34^{+}, \mathrm{CD} 45^{+}, \mathrm{CD} 14^{-}$. After cultivation with TNF $\alpha$ cocktail, the marker CD14 appeared in the mRNA preparation. The set of total mRNAs isolated and identified from such cells changed on $\mathrm{CD} 34^{+}, \mathrm{CD} 45^{+}, \mathrm{CD} 14^{+}$phenotype.

Since the work is largely concerned to exosomes, the results are presented in the form of a comparison of the properties of exosomes isolated from MSC and HSC cultures due to presence of sufficient information in the literature about exosomes from MSCs. Investigation of caspase activity as in HSCs isolated by immuno-affinity method, and in MSCs, was carried out with a non-peptide proteasome inhibitor to avoid misconceptions about the proteasomal protease involving. The proteasome inhibitors (bortezomib and $\mathrm{AdaAh}_{3} \mathrm{~L}_{3} \mathrm{VS}$ ) were used at the completely blocking concentrations of all protease activities of proteasomes. Data about proteasomal activities which are completely inhibited by bortezomib for different substrates are shown in Figure 1.

It should be noted that the activity was detected only after the addition of saponin, i.e., it is intracellular. Our attempts to induce apoptosis with cytochrome $C$ and dATP (under conditions of permeabilization of cells with saponin) were not effective in cases of HSCs or MSCs. We attempted to induce apoptosis with TNF $\alpha$ (for 4 days, adding it at a concentration of $10 \mathrm{ng} / \mathrm{mL}$ ) in culture with 
HSCs, but specific activity for caspases induced by cytochrome C and dATP was not detected also. In the $\mathrm{CD} 34^{+}$cell population, we also failed to detect intracellular protease activity selective for caspases induced by the addition of apoptosis inducers. Thus, it is possible to assume that MSCs and HSCs are not able to develop classical apoptosis induced by the release of cytochrome $\mathrm{C}$ from mitochondria.

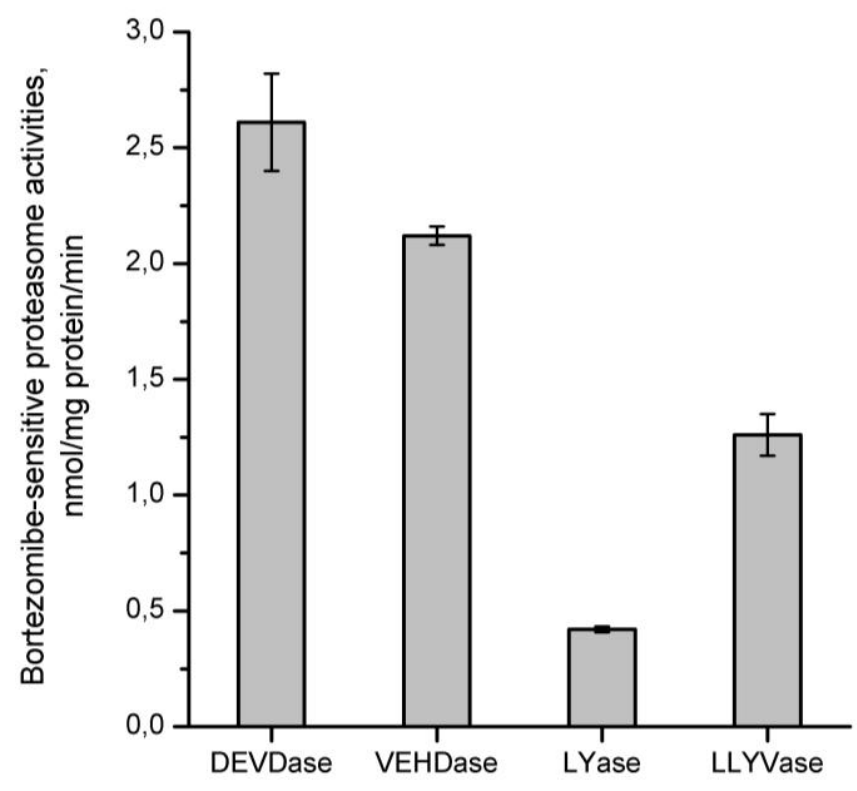

Figure 1. Activity of bortezomib-sensitive proteasomes in CD $34^{+}$HSCs. Data are presented as means $\pm \mathrm{SD}, n=3$.

At the same time, TNF $\alpha$-treated permeabilized CD34- cells had a little activity for the substrate (Z-DEVD)2R110, which was completely inhibited by the addition of $0.1 \mu \mathrm{M}$ emrikasan. In exosomes from such cells, saponin caused unmasking of caspase activity in vesicles (Figure 2). This also suggests the intracellular localization of active caspases in neutrophils and / or their precursors.

LLYVase activity that was inhibited by bortezomib and AdaAhx ${ }_{3} \mathrm{~L}_{3} \mathrm{VS}$ was present in exosomes (Figure 3). In the case of MSCs, this activity was manifested after the addition of saponin. Proteasomal activity in exosomes from HSC culture medium was in many folds lesser than in the case of MSCs and the addition of saponin did not increase this activity. At the same time, the activity in vesicles from the culture medium with TNF $\alpha$ cocktail was significantly higher than without it (Figure 3), but the activity after the addition of saponin did not increase. Also, this activity increases after treatment of probes with bovine serum albumin together with SDS, what is a characteristic feature of 20Sproteasomes (data not shown).

Chromatography of the concentrated medium from HSC culture showed that it contains proteasomes outside the exosomal membranes, because the peaks of proteasomal activity of samples prepared after treatment with Triton X-100 $(0.05 \%)$ corresponded to the profile of migration on the column of 205 proteasomes standard (Figure 4). The same profile was found for the specific caspase activity, which correlates with known data on the molecular size of apoptosomes, but the latter observation is true only for the use of TNF $\alpha$ cocktail during cultivation. No caspase activity was detected in the MSC culture medium. 


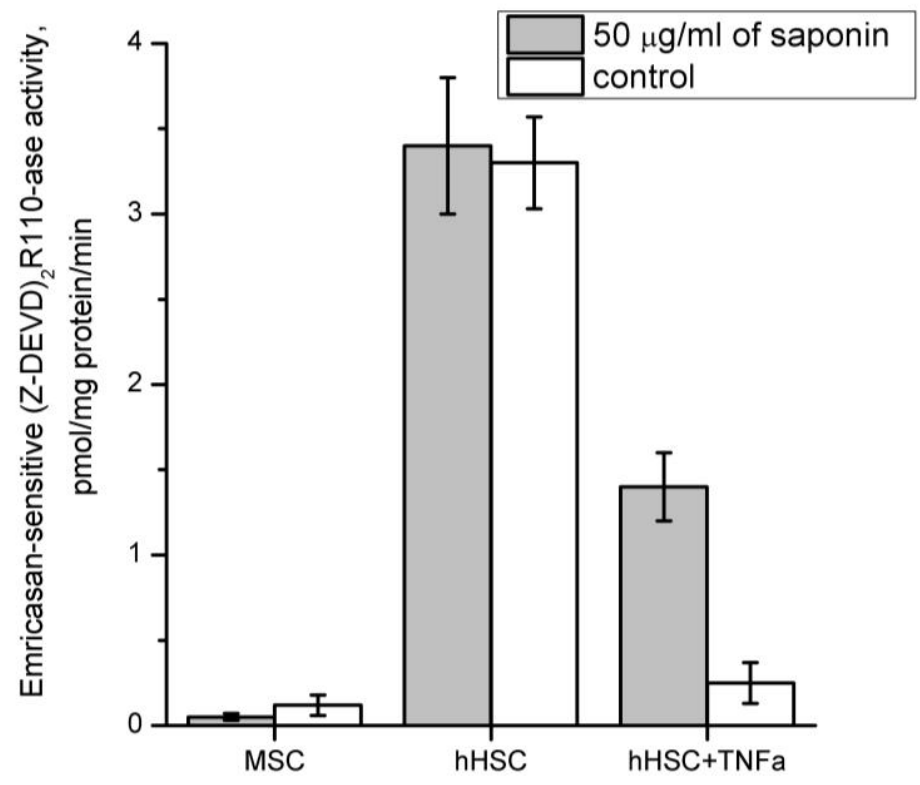

Figure 2. Activity of emricasan-sensitive (Z-DEVD) $)_{2}$ R110-ase in exosomes from HSC and MSC cultural medium. Data are presented as means $\pm \mathrm{SD}, n=3$.

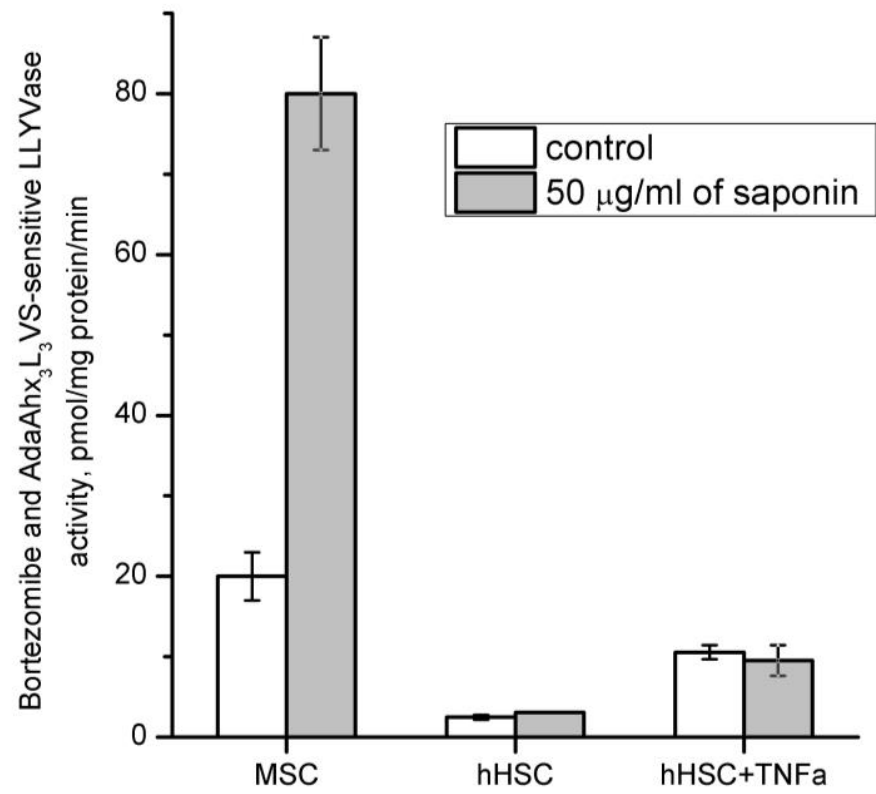

Figure 3. Bortezomib- and AdaAhx $x_{3} \mathrm{~L}_{3} \mathrm{VS}$-sensitive activity of LLYVase in exosomes from HSC and MSC cultural medium, in the presence and absence of saponin. Data are presented as means $\pm \mathrm{SD}, n$ $=3$. 


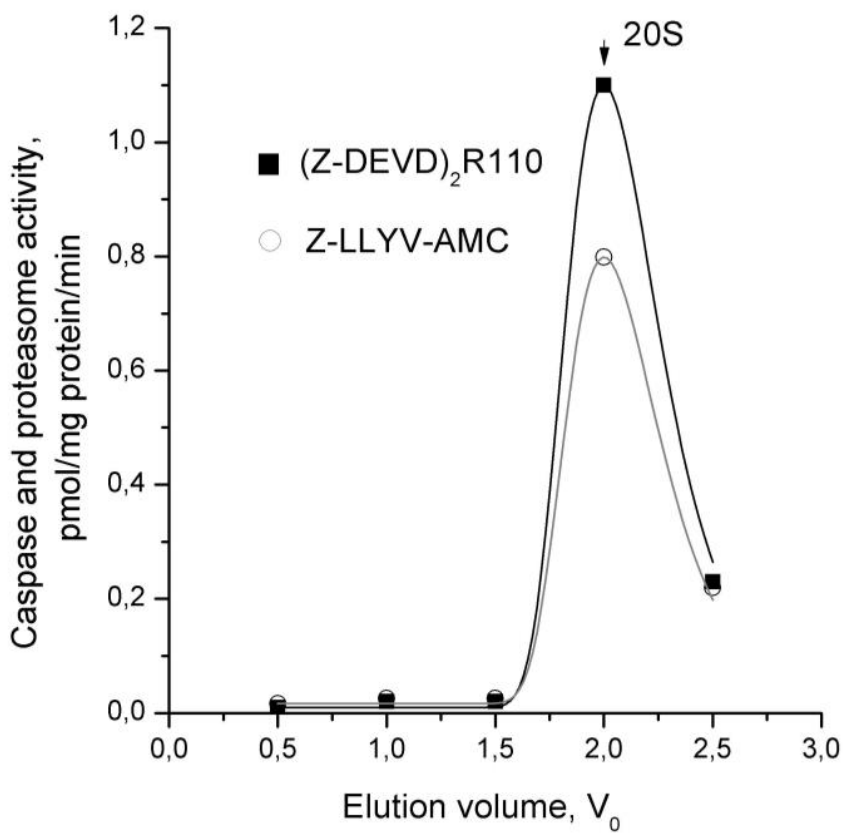

Figure 4. AdaAhx ${ }_{3} \mathrm{~L}_{3} \mathrm{VS}$ - and emricasan-sensitive activities of neutral proteases in the eluate during gel filtration of HSC cultural medium with TNF $\alpha$ Elution volume in free volume of the column $\left(\mathrm{V}_{\mathrm{o}}\right)$ is plotted on the $X$ axis. The arrow indicates the position of a standard sample of $20 \mathrm{~S}$ proteasomes (Enzo Inc., USA).

\section{Discussion}

We compared the exosomes' contents purified from conditioned culture medium of the adult HSCs and MSCs. It is known that therapeutic samples of exosomes generated by natural killers have a cytotoxic effect [24]. Apoptosis determination in cultivated cells and their extracellular vesicles can help to evaluate the cytotoxic potential of this possible medicine.

Commonly used methods of the apoptosis determination are include the identification of phosphatidylserine which appears on the outer part of the plasma membrane and binds annexin $\mathrm{V}$, the degradation of nuclear protein known as poly (ADP-ribose) polymerase, or the determination of enzyme activities that are should be distinguished from proteasome caspase-like activity [9]. In most of the early studies, the specificity of caspase activity was determined using peptide inhibitors. Simultaniously, the same substrates and inhibitors of caspases are the substrates and inhibitors of proteasomal caspase-like activity. They bind with the target enzymes in the same active center as the substrates, because they differ from the substrates by the aldehyde group at the C-terminus of the peptide only. This approach has promoted a lot of suggestions about the presence of apoptosis in adult stem cells. At the same time, experiments on turning off the critical genes for the apoptosis development did not correspond to this idea $[25,26]$. Also it should be taken into account that there are many variants of white blood cells death in humans - apoptosis, pyroptosis, necroptosis [10,11].

We revealed that secreted by HSCs exosomes give off proteasomes into the culture medium. Inhibitors used for the identification confirms that this activity relates to proteasome. High molecular weight filtration indicates that proteasomal activity presents in a $20 \mathrm{~S}$ proteasomes fraction. It is wellknown that proteasomes were found in extracellular vesicles of different body fluids [27-30]. We found that extravesicular proteasomal activity is also presented and its amount is quite comparable to intravesicular. This is also a known phenomenon, which is described for T-lymphocytes [2] and does not contradict the known observations about the presence of free, extracellular, intact and active proteasomes in plasma, lung fluid, cervical fluid, etc. [29,30].

The induction of apoptosome formation in MSCs and HSCs with cytochrome $\mathrm{C}$ was impossible and this correlates with the known data above. However, in the culture medium and in the exosomes, we detected caspase-like activity after the adding of the TNFa cocktail only. This activity is completely inhibited by a highly selective non-substrate inhibitor - emricasan, and is not sensitive to 
proteasome inhibitors. It should be noted that in this case, proteasome inhibitors, which are not structural analogues of their substrates was used, and this feature significantly increases the accuracy of the data. The detected caspase activity probably has two localizations - membrane and intracellular. This follows from data about activity after the permeabilization of membranes with saponin. Since in our work we used a cocktail of protease inhibitors (including serine proteases), it is possible to assume that caspase was activated during the spontaneous generation of differentiated leukocytes, and not during the permeabilization of membranes. The detected caspase activity is probably related to neutrophils whose membrane was decorated by active form of caspase- 3 which was preactivated by the PR3 protease [14]. Extracellular activity pertained to protein complexes of molecular weight similar to $20 \mathrm{~S}$ proteasomes and is probably represented by apoptosomes. Treatment of cell culture with TNF $\alpha$ results in the formation of intracellular apoptosomes that are given off from the exosomes (e.g., due to neutral sphingomyelidase activity). We assume that TNF $\alpha$ induces the generation of neutrophils and other leukocytes in culture of HSCs or the generation of other differentiated cells that are capable of apoptosis, in contrast to HSCs or MSCs [31-34].

Author Contributions: Prudnikov I., Smirnov A., Tsyvkin V. conceived and designed the experiments; performed the experiments; and analyzed the data; wrote the paper.

Acknowledgments: The reported study was funded by National Academy of Sciences of Ukraine according to the research project № II-1-20, 0120U001281.

Conflicts of Interest: The authors declare no conflict of interest. The founding sponsors had no role in the design of the study; in the collection, analyses, or interpretation of data; in the writing of the manuscript, and in the decision to publish the results.

\section{Abbreviations}

HSC: hematopoietic stem cell

MSC: mesenchymal stem cell

TNF $\alpha$ : tumor necrosis factor $\alpha$

PR3: serine proteinase 3

SERPINB1: serine protease inhibitor B1

MLKL: mixed lineage kinase domain-like protein

GSDMD: gasdermin D

SFEM: Serum-Free Expansion Medium

Flt3L: fms-related tyrosine kinase 3 ligand

SCF: stem cell factor

IL-2, -3, -6: interleukine $-2,-3,-6$

TPO: thrombopoietin

GM-CSF: granulocyte-macrophage colony-stimulating factor

(Z-DEVD)2R110: Z-Asp-Glu-Val-Asp)2-Rhodamine 110

AdaAhx ${ }_{3}{ }_{3}$ VS: Adamantane-acetyl-(6-aminohexanoyl) ${ }_{3}$-(leucinyl) ${ }_{3}$-vinyl-(methyl)-sulfone

Z-LLYV-AMC: Suc-Leu-Leu-Val-Tyr-7-amino-4-methylcoumarin

\section{References}

1. Prudnikov, I.M.; Tsyvkin, V.M.; Smirnov, A.M.; Pristash, L.V.; Chernyak, V.A.; Selyuk, V.M.; Muzichenko, P.F. Current comprehension of vesicular intercellular signaling. Fiziol. Zh. 2019, 65, 105-125, doi:https://doi.org/10.15407/fz65.06.105. Chernyak

2. Bochmann, I.; Ebstein, F.; Lehmann, A.; Wohlschlaeger, J.; Sixt, S.U.; Kloetzel, P.-M.; Dahlmann, B. T lymphocytes export proteasomes by way of microparticles: A possible mechanism for generation of extracellular proteasomes. J. Cell. Mol. Med. 2014, 18, 59-68, doi:10.1111/jcmm.12160.

3. Prudnikov, I.; Tsyvkin, V.; Smirnov, A.; Pristash, I.; Syrko, M. EXTRACELlULAR PROTEASOMES. Fiziolohichny̆ zhurnal 2020, 66, 73-84, doi:10.15407/fz66.05.073.

4. Ravichandran, R.; Bansal, S.; Rahman, M.; Sharma, M.; Liu, M.; Bharat, A.; Hachem, B.; Omar, A.; Smith, M.A.; Mohanakumar, T. The role of donor-derived exosomes in lung allograft rejection. Hum. Immunol. 2019, 80, 588-594, doi:10.1016/j.humimm.2019.03.012. 
5. Kitajima, Y.; Suzuki, N.; Nunomiya, A; Osana, S.; Yoshioka, K.; Tashiro, Y.; Takahashi, R.; Ono, Y.; Aoki, M.; Nagatomi, R. The Ubiquitin-Proteasome System Is Indispensable for the Maintenance of Muscle Stem Cells. Stem Cell Report. 2018, 11, 1523-1538, doi:10.1016/j.stemcr.2018.10.009.

6. Shan, H.; Li, X.; Xiao, X.; Dai, Y.; Huang, J.; Song, J.; Liu, M.; Yang, L.; Lei, Y.; Tong, Y. USP7 deubiquitinates and stabilizes NOTCH1 in T-cell acute lymphoblastic leukemia. Signal Transduct. Target Ther. 2018, 3, 29, doi:10.1038/s41392-018-0028-3.

7. Suresh, B.; Lee, J.; Kim, H.; Ramakrishna, S. Regulation of pluripotency and differentiation by deubiquitinating enzymes. Cell Death Differ. 2016, 23,1257-1264, doi:10.1038/cdd.2016.53.

8. Hao, Q.; Chen, Y.; Zhou, X. The Janus Face of p53-Targeting Ubiquitin Ligases. Cells 2020; 9, 1656, doi:10.3390/cells9071656.

9. Crawford, E.D.; Wells, J.A. Caspase substrates and cellular remodeling. Annu. Rev. Biochem. 2011, 80, 10551087, doi:10.1146/annurev-biochem-061809-121639.

10. Tanzen, V.; Fleming, H.E.; Riedt, T.; Karlsson, G.; Riese, M.J.; Celso, L.C.; Reynolds, G.; Milne, G.D.; Paige, C.J.; Reynolds, G. Hematopoietic stem cell responsiveness to exogenous signals is limited by caspase-3. Cell Stem Cell. 2008, 2, 584-594, doi:10.1016/j.stem.2008.03.012.

11. Wang, L.S.; Liu, H.J.; Xia, Z.B.; Broxmeyer, H.E.; Lu, L. Expression and activation of caspase-3/CPP32 in $\mathrm{CD}^{+}{ }^{+}$cord blood cells is linked to apoptosis after growth factor withdrawal. Exp. Hematol. 2000, 28, 907915, doi:10.1016/s0301-472x(00)00485-9.

12. Nonomura, K.; Yamaguchi, Y.; Hamachi, M; Koike, M.; Uchiyama, Y.; Nakazato, K.; Mochizuki, A.; SakaueSawano, A.; Miyawaki, A.; Yoshida, H. Local apoptosis modulates early mammalian brain development through the elimination of morphogen-producing cells. Dev. Cell. 2013, 27, 621-634, doi:10.1016/j.devcel.2013.11.015.

13. Ning, X.; Wang, Y.; Jing, M.; Sha M.; Lv, M.; Gao, P.; Zhang, R.; Huang, X.; Feng, J.-M.; Jiang, Z. Apoptotic Caspases Suppress Type I Interferon Production via the Cleavage of cGAS, MAVS, and IRF3. Mol. Cell. 2019, 74, 19-31, doi:10.1016/j.molcel.2019.02.013.

14. Loison, F.; Xu, Y.; Luo, H.R. Proteinase 3 and Serpin B1: A novel pathway in the regulation of caspase-3 activation, neutrophil spontaneous apoptosis, and inflammation. Inflamm. Cell. Signal. 2014, 1, e462, doi:10.14800/ics.462.

15. Burgener, S.S.; Leborgne, N.G.F.; Snipas, S.J.; Salvesen, G. S.; Bird, P. I.; Benarafa, C. Cathepsin G Inhibition by Serpinb1 and Serpinb6 Prevents Programmed Necrosis in Neutrophils and Monocytes and Reduces GSDMD-Driven Inflammation. Cell. Rep. 2019, 27, 3646-3656, doi:10.1016/j.celrep.2019.05.065.

16. Cao, M.; Chen, F.; Xie, N.; Cao, M.-Y.; Chen, P.; Lou, Q.; Zhao, Y.; He, C.; Zhang, S.; Song, X.; et al. c-Jun Nterminal kinases differentially regulate TNF- and TLRs-mediated necroptosis through their kinasedependent and -independent activities. Cell Death Dis. 2018, 9, 1-16, doi:10.1038/s41419-018-1189-2.

17. Xiao, Y.; Li, H.; Zhang, J.; Wei, W.; Breslin, P.; Zhang, J. TNF- $\alpha$ /Fas-RIP-1-induced cell death signaling separates murine hematopoietic stem cells/progenitors into 2 distinct populations. Blood 2011, 118, 60576067, doi:10.1182/blood-2011-06-359448.

18. Murphy, J.M.; Czabotar, P.E.; Hildebrand, J.M.; Lucet, I.S.; Zhang, J.G.; Alvarez-Diaz, S.; Lewis, R.; Lalaoui, N.; Metcalf, D.; Webb, A.I. The pseudokinase MLKL mediates necroptosis via a molecular switch mechanism. Immunity 2013, 39, 443-453, doi:10.1016/j.immuni.2013.06.018.

19. Samson, A.L.; Zhang, Y.; Geoghegan, N.D.; Gavin, X.J.; Davies K.A.; Mlodzianoski, M.J.; Whitehead, L.W.; Frank D.; Garnish, S.E.; Fitzgibbon C.; et al. MLKL trafficking and accumulation at the plasma membrane control the kinetics and threshold for necroptosis. Nat. Commun. 2020, 11, 3151, doi:10.1038/s41467-02016887-1.

20. Miao, E.A.; Leaf, I.A.; Treuting, P.M.; Mao, D.P.; Dors, M.; Sarkar, A.; Warren, S.E.; Wewers, M.D.; . Aderem, A. Caspase-1-induced pyroptosis is an innate immune effector mechanism against intracellular bacteria. Nat. Immunol. 2010, 11, 1136-1142, doi:10.1038/ni.1960.

21. Kayagaki, N.; Stowe, I.B.; Lee, B.L.; O’Rourke, K.; Anderson, K.; Warming S.; Cuellar T.; Haley B.; RooseGirma, M.; Phung Q.T.; et al. Caspase-11 cleaves gasdermin D for non-canonical inflammasome signaling. Nature 2015, 526, 666-671, doi:10.1038/nature15541.

22. Kedong, S.; Fan. X.; Liu. T.; Macedo, H.M.; Jiang L.; Fang M.; Shi, F.; Ma, X.; Cui, Z. Simultaneous expansion and harvest of hematopoietic stem cells and mesenchymal stem cells derived from umbilical cord blood. J. Mater. Sci. Mater. Med. 2010, 21, 3183-3193, doi:10.1007/s10856-010-4167-5. 
23. Prudnikov, I.M.; Smirnov, A.N. Short peptide tools for monitoring caspase and proteasome activities in embryonal and adult rat brain lysates: An approach for the differential identification of proteases. J. Biochem. 2012, 151, 299-316, doi:10.1093/jb/mvs001.

24. Batsali, A.K.; Georgopoulou, A.; Mavroudi, I.; Matheakakis, A.; Pontikoglou, C. G.; Papadaki, H. A. The Role of Bone Marrow Mesenchymal Stem Cell Derived Extracellular Vesicles (MSC-EVs) in Normal and Abnormal Hematopoiesis and Their Therapeutic Potential. J. Clin. Med. 2020, 9, 856, doi:10.3390/jcm9030856.

25. Singh, R.; Letai, A.; Sarosiek, K. Regulation of apoptosis in health and disease: The balancing act of BCL-2 family proteins. Nat. Rev. Mol. Cell. Biol. 2019, 20, 175-193, doi:10.1038/s41580-018-0089-8.

26. White, M.J.; McArthur, K.; Metcalf, D.; Lane R.M.; Cambier J.C.; Herold, M.J.; van Delft, M.F.; Bedoui,S.; Lessene, G.; Ritchie, M.E.; et al. Apoptotic Caspases Suppress mtDNA-Induced STING-Mediated Type I IFN Production. Cell 2014, 159, 1549-1562, doi:10.1016/j.cell.2014.11.036.

27. Lai, R.C.; Tan, S.S.; Teh, B.J.; Sze, S.K.; Arslan, F.; De Kleijn, D.P.; Choo, A.; Lim, S.K. Proteolytic Potential of the MSC Exosome Proteome: Implications for an Exosome-Mediated Delivery of Therapeutic Proteasome. Int. J. Proteom. 2012, 2012, 1-14, doi:10.1155/2012/971907.

28. Zoeger, A.; Blau, M.; Egerer, K.; Feist, E.; Dahlmann B. Circulating Proteasomes Are Functional and Have a Subtype Pattern Distinct from 20S Proteasomes in Major Blood Cells. Clin. Chem. 2006, 52, 2079-2086, doi:10.1373/clinchem.2006.072496.

29. Tsimokha, A.S.; Zaykova, J.J.; Bottrill, A.; Barlev, N.A. Extracellular Proteasomes Are Deficient in 195 Subunits as Revealed by iTRAQ Quantitative Proteomics. J. Cell. Physiol. 2017, 232, 842-851, doi:10.1002/jcp.25492.

30. Ramachandran, K.V.; Fu, J.M.; Schaffer, T.B.; Na, C.H.; Delannoy, M.; Margolis S.S. Activity-Dependent Degradation of the Nascentome by the Neuronal Membrane Proteasome. Mol Cell. 2018, 71, 169-177, doi:10.1016/j.molcel.2018.06.013.

31. Dong, T.; Liao, D.; Liu, X.; Lei, X. Using Small Molecules to Dissect Non-apoptotic Programmed Cell Death: Necroptosis, Ferroptosis, and Pyroptosis. Chembiochem. 2015, 16, 2557-2561, doi:10.1002/cbic.201500422.

32. Luo, H.R.; Am, F.L. Constitutive neutrophil apoptosis: Mechanisms and regulation. J. Hematol. 2008, 83, 288-295, doi:10.1002/ajh.21078.

33. Scheel-Toellner, D.; Wang, K.-Q.; Webb, P.R.; Wong S.H.; Craddock R.; Assi L.K.; Salmon M.; Lord J.M. Early events in spontaneous neutrophil apoptosis. Biochem. Soc. Trans. 2004, 32, 461-464, doi:10.1042/BST0320461.

34. Tang, R.; Xu, J.; Zhang, B.; Liu J.; Liang C.; Hua J.; Meng Q.; Yu X.; Shi S. Ferroptosis, necroptosis, and pyroptosis in anticancer immunity. J. Hematol. Oncol. 2020, 13, 110, doi:10.1186/s13045-020-00946-7.

Publisher's Note: MDPI stays neutral with regard to jurisdictional claims in published maps and institutional affiliations.

(C) 2020 by the authors. Licensee MDPI: Basel, Switzerland. This article is an open access article distributed under the terms and conditions of the Creative Commons Attribution (CC BY) license (http://creativecommons.org/licenses/by/4.0/). 